$4^{\circ}$ Effectuer la détermimation de l'acidité aussi rapidement que pos sible, car les pellicules, en perdanl de leur hunidité, changent quelque peu de teinte; la célérité est donc recommandable, mime avec les modèles perfectionués de curseur munis d'un courercle retardant quelque peu la dessication.

Comme preuve de l'exactitude ct de la commodité de la méthode, donnons cet exemple diun lait de cham fràchement préparé, réaction fortement basique ( $p H=9$ ) utilisé pour le traitement d'un étang nettement acide $(p H=5,6)$. Après l'opération, une alténuation notable de l'acidité fut enregistrée, le degré de concentration ayant été trouvé ég̣al à 6,3 .

Par ailleurs, voici plus de deux ans quie nous employons cette méthode d.: foliocolorimètre pour les recherches portant sur les exploitations d'élangrs silésiens et elle nous a domé la plus complète satislaction.

Très sensible, elle fournit, sur la qualité de l'eau, des renseignements aussi précis que besoin en ext pour sori contròle et permet de discemer toutes les modifications se produisant daus un étangr, comme conséquence des variations de la concentration.

On sait, aujourd hui, que pour heaucoup) de parasites aqquatiques, l'éclo-

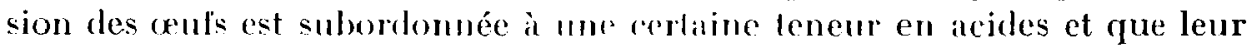
multiplication ou leur morlalité r: masse sont dépendanles de la réaction de l'eau. De mème, les divers animaux su végrtaux d'un étang prospèrent au mieux pour une valeur définie de $p \mathrm{H}$.

Par chaulage, ou par apport d'autres engrais artificiels, on peut exercer une influence directrire sur la vie limnique, du moment où l'on dispose d'un moyen simple al précis, comme celui qui vient d'ùtre décrit, pour contraindre les élangrs à révéler quelque chose dè leur secret.

\title{
LA VFNTE DU POISSON SUR LA BONDE
}

\section{Par le Comte Georcies LE COINTRE}

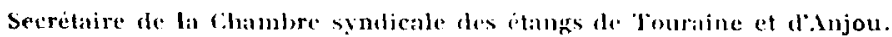

Il suffit de voir le public se presser aux abords des aquariums d'unc exposition piscicole pour se rendre compte de l'altrait qu'exerce sur la foule la gent aquatique. Iltrait de mystère ?... peut-ètre?

Nos Poissons, bien qu'en quelque sorte demestiqués par une pisciculture savante ne se voicnt pas aussi fréfuemment à l'état visant que Poules, Lapins et Cochons et peut dite cela coplique-t il lat curiosile quexcite une fêche d'étang. Il faut avoir vu, aulour d'une pècherie, les centaines de regards comme hypotisés par la bouche jaune des Carpes, le scintillement du Brochet, la souple sinuosité de l'Intruille ! Aussi n'est-il pas difficile, avec un peu de publicité, de grouper quelques centaines, voire 
même quelques milliers de personnes autour d'une bonde d'étang en pêchè. Naturellement, avant de rentrer, elles voudront emporter comme souvenir qui une belle Carpe, Anguille ou Brochet, qui un petit panier de friture. Et c'est assez pour permettre d'écouler quelqunes centaines ou quelques milliers de kilos de Poisson, dont la presque totalité dé la " friture " que tout étang ne manque pas de produire.

Mais, me direz-vous, nous ne sommes pas marchands de profession. Comment nous y prendre?

Pour vous bien répondre, il me faudrait ittre chez vous, avec voưs. Ici je me bornerai donc à vous donner quelques directives, îruit de mon expérience personnelle. Si vouz voulez des détails, venez voir contment se parse une de ces ventes. Aucum article de rovue ne peut remplacer l'expérience de visu et, d'ailleurs les royagres forment la jeunesse - et même l'âge mâr, - en pisciculture comme en toutc autre matière.

Une condition essentielle de la réussite sst que le personnel puisse travailler en toute tranquillité à l'abri de toute ingérence on bousculade de la part du public. Il est non moins nécessaire que re dernier puisse voir tout ce qui se passe, car il vient là encore plus pour voir que pour acheter.

Pour ma part, j'ai résolu ce double problème en construisant une cloture continue englobant la pêcherie, la cabane de vente et, entre les deux, un espace suffisant pour permettre les diverses manipulations du yoisson. La balustrade d'enceinte permet au public de s'accouder le long d'un des côtés de la pêcherie, l'autre étant réservé au service. Un autre côté de l'enclos est longé par le couloir où les acheteurs fónt la queue devant la cabane de vente, ce qui leur permet de se distraire en regardant les opérations de tri et de pesée. Le troisième côté longe le bas du talus de la bonde et permet au public de s'ćtacer comme dans un amphithéàtre pour ne rien perdre du spectacle. Face à la cabane de. vente sont installés les forains : marchands de marrons, de trâteaux, d'huîtres, buvette, etc., chauffés par un grand brasier de fagots.

Voici, maintenant, ce qui se passe à l'in'éricur de l'enclos : deux pêcheurs, chaussés de hautes botles cuissardes en caoutchouc et armés d'épuisetles, descendent tour à tour dans la pècherie, l'un prend le Poisson et passe l'épuisette à son camarade, resté au dehors, qui la vide sur la täble de triage. De là, le Poisson classé par catégories, est porté dans des cageots entoilés, de tare uniforme, sur une basculle auprè̀s de laquelle un homme de confiance en registre les pesées et dirige le Poisson sur sa destination (réserve, expédition, vente au détail, etc.). Le Pơisson destiné à la vente au détail est porté à la cabane où les vendeurs le détaillent au moyen d'une balance Roberval dont un des plateaux a été remplacé par un panier ou une hoîte en tile perforée. Le tarif est affiché en vue des acheteurs et les vendeurs ont, de leur côté, un barème donnant, selon la pesée qui va de livre en livre, la somme à réclamer. Donc, aucun calcul et la rapidité est extrême. Pour bien faire, il faut, par balance, 
deux vendeurs; lun conserve ses mains propres, calcule et encaisse : l'autre manipule le Poisson, le pèsé et le remet au client. La seule difficulté réside dans le fait que, souvent, les spectateurs, captivés par le spectacle de la pêcherie se décident à faire leurs emplettes que quelques instants avant de s'en aller. Il en résulte un "coup de feu "pendant lequel les vendeurs risquent de sc trouver débordés. On peut parer à cela en mettant en service, à ce moment, une balance supplémentaire, servie, par exemple, par le personnel de la bascule.

En résumé, pour un étang de g liectares, donnant linoo kilogrammes de Poisson varié, j'emploie? pècheurs, i peseur (le garde), a trieurs (souvent des femmes), 2 manouvres, ? vendeurs. Grâce à la clôture, le personnel travaille à son aise, le public voit tout, mais n'a d'accès au Poisson que par la cabane de vente dans laquelle il est contenu par une table longue qui le sépare des vendeurs. La surveillance est donc très facile. Le contrôle de la vente est assuré de façon inutonaligue par la pesée en $\because$ ros de tout ce qui entre dans la cabane. Avec cette organisation, le

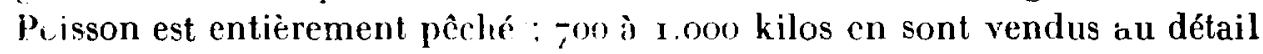
airisi que quelques centaines de kilos en demi-gros aux revendeurs locaux, tout cela avant midi. On dispose de toute la soirée pour parachever la vonte au détail,, diriger le reste du Poisson aux réserves ou. vers la gare et ranger le matériel, ce qui n'est pas la partie la moins importante de l'opération.

Il va de soi que l'organisation varie suivant l'étang et les conditions locales. Je connais tels sociétaires qui vendent au détail 4.000 kilos de Poisson le premier jour de la pîche; il faut à ceux-ci 8 balances et du personnel en proportion.

Ce n'est pas la première fois que je conseille ce mode de vente à mes rollègues de la Chambre syndicrl!' de Tr,uroine et d'Aninu. D'aucuns m'ont écouté et s'en sont hien trouvés ; d'autres m'ont répondu : "Tout ça fait bien du tracas, nous aimerions bien mieux aller au cinéma ou i la chasse pendant la pêche et charger la Chambre syadicale de tout faire... "Il y en eut mème un qui préférait mème ne pas empoissonner son f́tang pour.. n'avoir pas "l'ennui ") de le picher!

\title{
LIMITES DE GROISSANGE
}

\author{
Par le Comte de NEUfBOURG
}

Nous avons observé, depuis trois ans, les résultats suivants sur la Carpe Meurniot-Hirsch, c'est-à-dire croisće de Gálicienne (Franconienne WaIderdorff) et de Bohémienne (Willinģau Schwartzenberğ), dans nos étangs dè Beauvoir, en Forez, culture normale sans nourriture. 